\title{
Optical Coherence Tomography Findings in a Case of Unilateral Acute Idiopathic Maculopathy
}

\author{
José Andonegui*, José Ramón Maya, Ane Zurutuza, Mónica Pérez de Arcelus
}

Ophthalmology Department, Complejo Hospitalario de Navarra, Pamplona, Spain.

Email: jandonen@cfnavarra.es

Received July $8^{\text {th }}, 2012$; revised September $18^{\text {th }}, 2012$; accepted October $5^{\text {th }}, 2012$

\begin{abstract}
Purpose: To describe the clinical, angiographic, and Optical Coherence Tomography (OCT) findings in a patient with Unilateral Acute Idiopathic Maculopathy (UAIM) and to discuss a possible pattern of OCT findings in patients with this disease. Methods: Case report. Results: A 34-year-old man with UAIM was evaluated. During the acute phase fundus examination revealed a yellow thickening in the macular area of the right eye, Fluorescein Angiography (FA) showed early alternating patches of hypofluorescence and hyperfluorescence and late hyperfluorescence and OCT showed thickening of the outer retina in the foveal zone. Six weeks later, the macula had pigmentary mottling, FA showed an area of central hypofluorescence surrounded by a hyperfluorescent ring that remained unchanged during the angiogram and the OCT examination was normal. Conclusion: OCT is a useful diagnostic tool for UAIM. OCT evaluation of patients with this disease will exhibit the heterogeneous hyperreflectivity and thickening of the outer retina and Retinal Pigment Epithelium (RPE). A serous macular detachment may not be a prominent finding in UAIM.
\end{abstract}

Keywords: Unilateral Acute Idiopathic Maculopathy; Optical Coherence Tomography

\section{Introduction}

Unilateral Acute Idiopathic Maculopathy (UAIM), a rare clinical entity of unknown etiology, affects otherwise healthy young adults who present with sudden loss of central vision sometimes preceded by a viral illness or flulike symptoms. Patients usually experience complete spontaneous resolution without treatment within few weeks after the onset [1,2]. Since the initial description of this disease by Yanuzzi et al. in 1991, very few cases have been reported in the ophthalmic literature, and most of these cases were reported before the introduction of Optical Coherence Tomography (OCT) as a routine tool for diagnosing retinal pathology. We describe the clinical, angiographic, and OCT findings in a patient with UAIM. A possible pattern of OCT findings in patients diagnosed with UAIM is discussed.

\section{Case Report}

A 34-year-old man presented with a 4-day history of Visual Acuity (VA) loss in his right eye. A few days previously he had had an upper respiratory tract infection. The VA was 0.2 in his right eye and 1.0 in his left eye. The anterior segment examination was unremarkable bilaterally. The fundus examination revealed a yellow

*Corresponding author. thickening in the macular area of the right eye (Figure 1) and a normal macula in the left eye. Fluorescein Angiography (FA) showed early alternating patches of hypofluorescence and hyperfluorescence and late hyperfluorescence in the right eye (Figure 2) and a normal left eye. OCT showed thickening of the outer retina in the foveal zone of the right eye (Figure 3); the left eye was unremarkable. A diagnosis of UAIM was established and no treatment was initiated.

Six weeks later, the VA returned to 1.0 in the right eye. The macula had pigmentary mottling (Figure 1). FA showed an area of central hypofluorescence surrounded by a hyperfluorescent ring that remained unchanged during the angiogram (Figure 2). The OCT examination was normal (Figure 3).

\section{Discussion}

UAIM is an inflammatory process involving the outer retina and Retinal Pigment Epithelium (RPE). The condition is considered to be idiopathic, although a possible association with Coxsackie virus was hypothesized [3]. In most cases, the involvement is unilateral, but bilateral lesions have also been reported [2,4].

The diagnosis of UAIM had been previously based on fundus examination and FA findings. More recently, OCT was introduced as a diagnostic tool in this entity. 


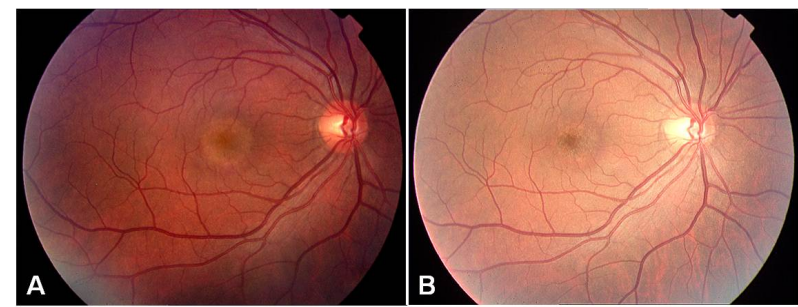

Figure 1. (A) Yellow thickening of the macular area is seen in the acute disease phase; (B) Pigmentary mottling of the macula is seen 6 weeks after onset.

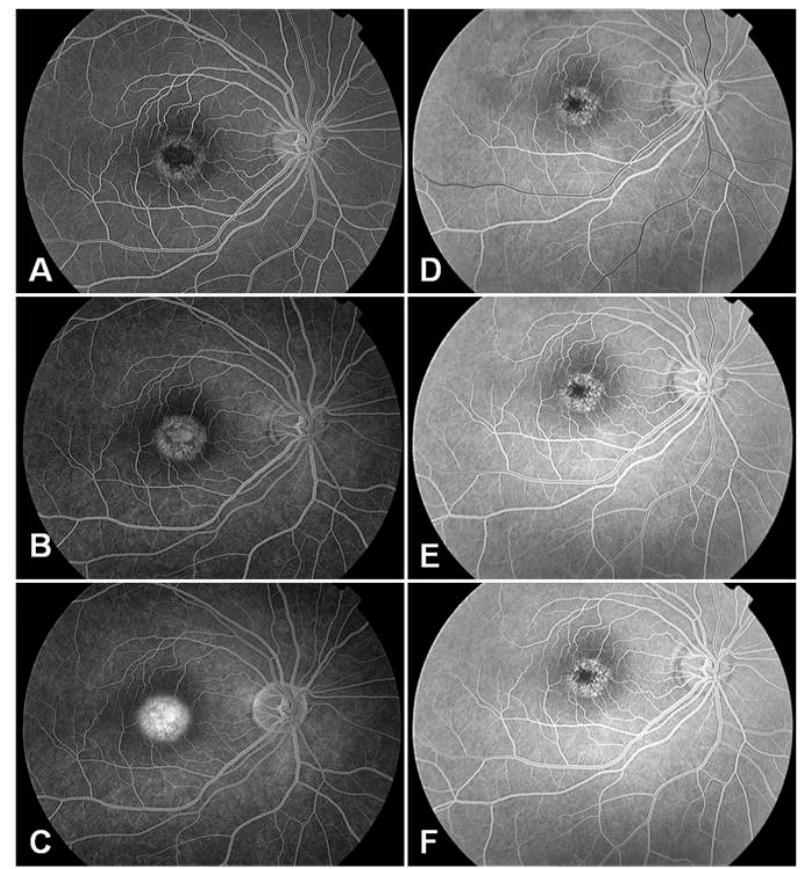

Figure 2. FA in the acute phase (A-C) and 6 weeks after (D, E). (A) Early phase of the angiogram showing a central area of hypofluorescence and a peripheral ring of hyperfluorescence; (B) A progressive increase of the fluorescence is seen; (C) A late-phase of the angiogram shows marked hyperfluorescence; (D) An early-phase angiogram revealing a central hypofluorescent area surrounded by a hyperfluorescent ring; $(E, F)$ The angiographic pattern remains unchanged in the late phases.

Regarding the clinical findings on fundus examinations, a serous neurosensory detachment with an irregular white, yellow, or gray thickening of the overlying RPE has been typically described in most patients [1,2]. Papillitis, subretinal exudation, intraretinal hemorrhages, or vitreous cells have also been observed [2]. After resolution, pigment epithelial atrophic changes and irregular pigmentation in the area of the initial macular involvement can be detected.

Angiographic findings in UAIM have been reported as early irregular hyperfluorescence alternating with areas of hypofluorescence and late complete staining of the lesion in the acute disease phase [1,2]. These angio-
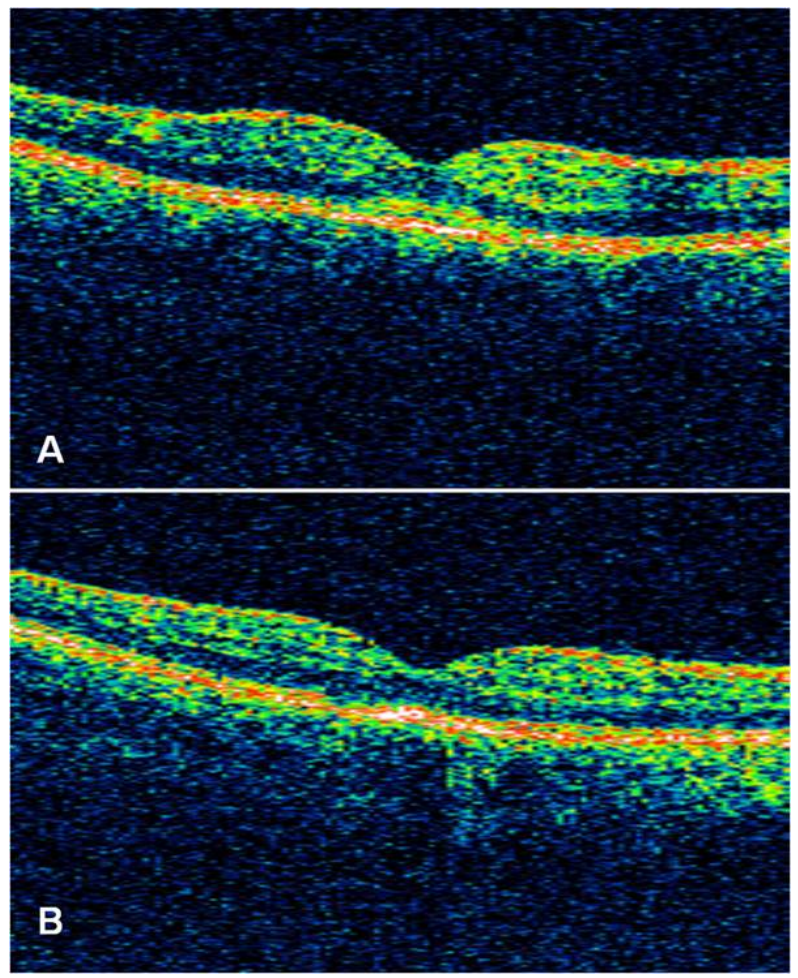

Figure 3. (A) An OCT image in the acute phase showing hyperreflectivity and thickening of the outer retina in the foveal zone; (B) The OCT image is unremarkable 6 weeks later.

graphic changes have been considered to be similar to the angiographic features of occult choroidal neovascular membranes [1]. Once improvement has occurred, the angiographic pattern appears as a central area of hypofluorescence due to blockage by the RPE hyperpigmentation surrounded by a hyperfluorescent zone of RPE atrophy. This angiographic configuration corresponds to a bull'seye maculopathy.

The descriptions of OCT findings in UAIM in the ophthalmic literature have been scanty and heterogeneous. A Medline search identified only seven cases of UAIM evaluated using OCT [4-10]. Gupta et al. [5] reported a patient with a prominent serous macular detachment, but the fluorescein angiogram of this patient was not characteristic of UAIM, because it lacked the characteristic early hypofluorescence and hyperfluorescence and late hyperfluorescence described in this condition. Ghazi et al. [4] also described an atypical case characterized by a macular hole in one eye and a small extrafoveal serous detachment of the neurosensory retina in the fellow eye. This case also lacked the hyperfluorescence pattern in the angiogram. Another case with serous detachment reported by $\mathrm{Xu}$ et al. [6] also presented an atypical angiogram and the atypical feature of recurrence of the disease. Only the patient reported by Haruta et al. [7] had a serous detachment on OCT and a typical angiographic 
pattern. The patients reported by Aggio et al. [8], de la Fuente et al. [9] and Ooto et al. [10] closely resembled the current patient. These patients exhibited the typical clinical and angiographic features of UAIM, but no subretinal fluid was seen on the OCT images.

OCT has helped to achieve a better understanding of many retinal diseases including UAIM. This entity consists of an inflammatory process of unknown etiology involving the outer retina and RPE that breaks down the inner or outer hemato-retinal barriers and intraretinal exudation in the outer retina responsible for the visual loss, the hyperfluorescence observed in FA, and the thickening detected on OCT. According to the results of the current OCT evaluation and the ones described by Aggio et al. [8] de la Fuente et al. [9] and Ooto et al. [10], we hypothesized that a neurosensory macular detachment is not a prominent feature of UAIM. All these were typical cases of UAIM, and in all of them OCT showed heterogeneous hyperreflectivity and thickening of the outer retina and RPE without a sensory macular detachment. Before introduction of OCT, a neurosensory macular detachment was reported in most patients with UAIM. However, a serous macular detachment can be difficult to detect during a fundus examination unless it is very prominent. FA in these patients characteristically showed early irregular hypofluorescence and hyperfluorescence followed by late hyperfluorescence of the entire lesion. This angiographic pattern is more characteristic of an exudative process of the outer retina than of a serous macular detachment, characterized by smooth early hyperfluorescence with a slight increase in the late phases.

We concluded that OCT is useful for diagnosing UAIM. Even thought more cases are needed to confirm our hypothesis, we believe that an OCT evaluation of patients with this disease will exhibit the heterogeneous hyperreflectivity and thickening of the outer retina and RPE. A serous macular detachment may not be a prominent finding in UAIM.

\section{REFERENCES}

[1] L. A. Yanuzzi, L. M. Jampol, M. F. Rabb, J. A. Sorenson, C. Beyrer and L. M. Wilcox, "Unilateral Acute Idiopathic
Maculopathy,” Archives of Ophthalmology, Vol. 109, No. 10, 1991, pp. 1411-1416. doi:10.1001/archopht.1991.01080100091049

[2] K. B. Freund, L. A. Yanuzzi, G. R. Barile, R. F. Spaide, S. A. Milewski and D. R. Guyer, "The Expanding Clinical Spectrum of Unilateral Acute Idiopathic Maculopathy,” Archives of Ophthalmology, Vol. 114, 1996, pp. 555-559. doi:10.1001/archopht.1996.01100130547007

[3] A. P. Beck, L. M. Jampol, D. A. Glasser and J. S. Pollack, "Is Coxsackievirus the Cause of Unilateral Acute Idiopathic Maculopathy," Archives of Ophthalmology, Vol. 122, No. 1, 2004, pp. 121-123. doi:10.1001/archopht.122.1.121

[4] N. G. Ghazi, A. Daccache and B. P. Conway, "Acute Idiopathic Maculopathy. Report of a Bilateral Case Manifesting a Macular Hole,” Ophthalmology, Vol. 114, No. 5, 2007, pp. e1-e6. doi:10.1016/j.ophtha.2006.08.055

[5] A. Gupta, S. Rogers and B. N. Matthews, "Unilateral Acute Idiopathic Maculopathy,” British Journal of Ophthalmology, Vol. 93, No. 8, 2009, pp. 1073-1074. doi:10.1136/bjo.2008.140335

[6] H. Xu and P. Lin, "Unilateral Recurrent Acute Idiopathic Maculopathy," Graefe's Archive for Clinical and Experimental Ophthalmology, Vol. 249, No. 6, 2011, pp. 941944. doi:10.1007/s00417-010-1589-1

[7] H. Haruta, M. Sawa, Y. Saishin, N. Ohguro and Y. Tano, "Clinical Findings in Unilateral Acute Idiopathic Retinopathy. New Findings in Acute Idiopathic Maculopathy,” International Ophthalmology, Vol. 30, No. 2, 2010, pp. 199-202. doi:10.1007/s10792-009-9299-6

[8] F. B. Aggio, M. E. Farah, R. L. Meirelles and E. C. de Souza, "Stratusoct and Multifocal ERG in Unilateral Acute Idiopathic Maculopathy," Graefe's Archive for Clinical and Experimental Ophthalmology, Vol. 244, No. 4, 2006, pp. 510-516. doi:10.1007/s00417-005-1172-3

[9] M. A. de la Fuente and R. Cuadrado, "Unilateral Acute Idiopathic Maculopathy: Angiography, Optical Coherence Tomography and Microperimetry Findings," Journal of Ophthalmic Inflammation and Infection, Vol. 1, No. 3, 2011, pp. 125-127. doi:10.1007/s12348-010-0014-6

[10] S. Ooto, M. Hangai and N. Yoshimura, "Photoreceptor Restoration in Unilateral Acute Idiopathic Maculopathy on Adaptive Optics Scanning Laser Ophthalmoscopy," Archives of Ophthalmology, Vol. 129, No. 12, 2011, pp. 1633-1635.doi:10.1001/archophthalmol.2011.345 